\title{
Using Social Choice Function Vs. Social Welfare Function To Aggregate Individual Preferences In Group Decision Support Systems
}

Dat-Dao Nguyen, California State University, Northridge, USA

\begin{abstract}
In multi-criteria decision making, any Group Decision Support System (GDSS) requires a "social judgment model" for calculation of weights on decision alternatives, and tabulation of individual votes toward a consensus. One could assess a Social Welfare Function - such as Keeney's - to aggregate individual cardinal preferences or utilities into a group preference. Alternatively, one could use Social Choice Functions - such as Condorcet, Borda, Copeland, and Eigenvector - to aggregate individual ordinal preferences or rankings into a group ranking. This study empirically investigates the consensus between individual preferences and the group preference derived from various aggregation methods.
\end{abstract}

Keywords: Social Welfare Function; Social Choice Function; Group Decision Support System

\section{INTRODUCTION}

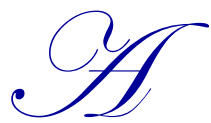

ny Group Decision Support System (GDSS) requires a "social judgment model" for calculation of weights for decision alternatives, and tabulation of individual votes toward a consensus in a multicriteria decision making setting (DeSanctis et al., 2008; Turban et al., 2011). One can achieve such consensus with a social welfare function - a group utility function (Keeney \& Kirkwoods, 1975; Keeney, 1976) to aggregate individual cardinal preferences or utilities into a group preference. The difficulty is in assessing the weights of individual judgments and trade-offs among individual utilities. An alternative is to aggregate individual ordinal preferences, or rankings, into a group ranking with social choice functions such as Condorcet, Borda, etc ... (Richelson, 1975, 1978a, 1978b, 1981) to count the votes of group members.

This study empirically investigates the consensus between individual preferences and the group preference derived from various aggregation methods. It highlights a comprehensive rule for the effective aggregation of individual preferences into a high group consensus.

\section{SOCIAL WELFARE FUNCTION APPROACH FOR GROUP PREFERENCE}

A social welfare function aggregates individual preferences into a group preference. Keeney and Kirkwoods (1975) and Keeney (1976) proposed that a group is an entity whose decisions obey the axioms of utility theory, then they formulated a group cardinal utility function.

For each alternative $a_{\mathrm{j}}, j=1,2, \ldots, m$, there exists an individual cardinal utility $u_{\mathrm{i}}\left(a_{\mathrm{j}}\right)$ for individual $I_{\mathrm{i}}, i=1$, $2, \ldots, n$. These individual utilities could be assessed by a procedure described in Keeney (1977).

Then the group cardinal utility for alternative $a_{\mathrm{j}}$ is:

$u_{\mathrm{G}}=u\left(u_{1}, u_{2}, \ldots, u_{\mathrm{n}}\right)$ 
This function could be in additive form:

$u_{\mathrm{G}}=\sum_{\mathrm{i}=1}^{\mathrm{n}} k_{\mathrm{i}} u_{\mathrm{i}}$

or in multiplicative form:

$1+k u_{\mathrm{G}}=\sum_{\mathrm{i}=1}^{\mathrm{n}}\left[1+k k_{\mathrm{i}} u_{\mathrm{i}}\right]$

where $k$ and $k_{\mathrm{i}}$ are scaling constants with $0<k_{\mathrm{i}}<1 ; k>-1$ is a non zero constant and is the solution to:

$1+k=\sum_{\mathrm{i}=1}^{\mathrm{n}}\left[1+k k_{\mathrm{i}}\right]$

$k_{\mathrm{i}}$ can be interpreted as the weight for individual utility. It is the marginal rate of trade-off in individual utility to increase the utility of other members. The sign of $k$ implies whether individual utilities are substitute or complementary.

The process requires interpersonal comparisons of utilities by a benevolent dictator or by a participatory group (Keeney \& Kirkwoods, 1975; Keeney, 1976). The benevolent dictator - someone has responsibility for the decision but wishing to take into account the views of others - assesses and decides on the weights of individual utilities (Keeney, 1976). In a participatory group, all members agree upon an aggregation rule for selecting the best alternative.

Preference is a highly individual matter as people may differ considerably in their judgments and expressions. Consequently, a group utility should be an aggregation of individual utilities/preferences based on a specified aggregating rule rather than a direct assessment of collective judgment (Wallsten \& Budescu, 1983).

The trade-offs of individual utilities require the interpersonal comparisons of utilities and the relative weights given to individual preferences. This process involves political issues, even ethical issues, in distributing power to group members. One also has the difficulty in deciding whether the model should be additive or multiplicative; i.e., whether individual utilities are substitute or complementary. Then trade-offs among member weights have to be reassessed when the group changes its members.

In a group decision, one may not expect the consensus on value or utility of each element of the decision problem. There is a distinction between consensus of values and consensus of choices. Achieving unanimity on values of attributes is not expected and it is usually not necessary for members to agree on some choices of alternatives (Ben-Arieh \& Easton, 2007).

\section{SOCIAL CHOICE FUNCTION APPROACH FOR GROUP PREFERENCE}

A comprehensive alternative is to derive a group ranking based on individual rankings/ordinal preferences. This social choice function approach uses a counting rule to aggregate individual ranking of alternatives/candidates or individual votes into a group ranking (Richelson, 1975, 1978a, 1978b, 1981.)

A voting procedure can be differentiated into either a non-ranked or a preferential system (Hwang \& Lin, 1987). In non-ranked or spot voting, a decision maker specifies only the most desirable candidate. Since opinions on the respective merits of other candidates are not given, this judgment set is incomplete. In preferential voting, the decision maker indicates the order of preference in which he/she would place all the candidates.

The study reported herein experiments with preferential voting process and the aggregation of individual rankings of candidates into a group choice using social choice functions.

Define:

- $\quad x>y$ as $x$ is preferred to $y$; 
- $\quad \#\left(i: x>_{\mathrm{i}} y\right)$ as the number of members who preferred $x$ to $y$;

- $y \in \mathrm{A} /\{x\}$ as $y$ is belonging to the set of alternatives $A$ in which $x$ is excludes from $A$

\section{Condorcet Function:}

$f_{\mathrm{C}}=\min \#\left(i: x>_{\mathrm{i}} y\right) \quad \mathrm{y} \in \mathrm{A} /\{\mathrm{x}\}$

This function measures the lowest number of votes in favor of against any other candidate. The Condorcet function elects the candidate having the largest minimum votes against the others

\section{Borda Function:}

$f_{\mathrm{B}}=\sum \#\left(i: x>_{\mathrm{i}} y\right) \quad y \in A$

The Borda score of a candidate $x$ is equivalent to the total number of group members who have $x$ preferred to $y$ for all $y$ in the set of alternatives under consideration. The candidate with the highest Borda score is the winner.

\section{Copeland Function:}

$f_{\mathrm{CP}}=\#(y: y \in A$ AND $x>y)-\#(y: y \in A$ AND $y>x)$

This function measures the number of candidates in $A$ that $x$ has a strict simple majority over, minus the number of candidates in $A$ who have strict simple majority over $x$. The candidates are ranked according to the value of $f_{\mathrm{CP}}$.

\section{Eigenvector Function:}

Let $x_{\mathrm{i}}, x_{\mathrm{j}} \in A$ and $n_{\mathrm{ij}}$ be the number of voters that have the alternative xi preferred to the alternative $x_{\mathrm{j}}$. Let $\mathbf{D}$ be a pairwise comparison matrix with elements defined as follows:

$d_{\mathrm{ij}}=\left(n_{\mathrm{ij}} / \mathrm{n}_{\mathrm{ji}}\right) \quad i \neq j ; d_{\mathrm{ji}}=1 / d_{\mathrm{ij}} i \neq j ; d_{\mathrm{ii}}=1$

The element $d_{\mathrm{ij}}$ shows the relative strength of preference for each pair of alternatives. This is a reciprocal matrix having all positive values. The eigenvector associated with the largest positive eigenvalue of matrix $\mathbf{D}$ will give the relative preference that the group assigned to each alternative.

A group ranking based on ordinal preferences may produce ties. In this case, one has to use linear programming techniques, such as "Minimum Variance" (Cook \& Seiford, 1982), to derive a compromised solution. However, the social preference ordering will not contain any tie if the number of voters is odd and voters are nor allowed to express indifference. In case of intransitivity, another subsidiary principle is needed to get a solution from the cycles; e.g., "Game-Theoretic Procedure" (Felsenthal \& Machover, 1992). In fact, these authors also noted that a considerably lower prevalence of cycles was found in empirical investigations than being predicted in theoretical studies.

\section{MEASURE OF GROUP CONSENSUS}

The group consensus is measured with the Kemeny function (Kemeny \& Snell, 1962.) This function is classified as an eminently fair, reasonable social choice function among top satisfactory functions with respect to conditions proposed in the literature (Levenglick, 1975; Richelson, 1981.) The Kemeny function finds the maximum total amount of agreement or similarity between the consensus ranking and individual preference orderings on the set of alternatives A.

Let a group of $n$ members rank a set of $m$ alternatives. 
Definition 1: Let $\mathbf{L}=\left(l_{\mathrm{ij}}\right)$ be the ranking matrix. The element $l_{\mathrm{ij}}$ can take the following values: 1 if $a_{\mathrm{i}}>a_{\mathrm{j}} ; 0$ if $a_{\mathrm{i}} \sim a_{\mathrm{j}} ;-1$ if $a_{\mathrm{i}}<a_{\mathrm{j}}$; ;here $\mathrm{i}, \mathrm{j}=1,2, \ldots, m$ is the index of alternative $a_{1}, a_{2}, \ldots, a_{\mathrm{m}}$. This matrix $\mathbf{L}$ is derived from any possible linear order of $A$, for example, the order obtained from a particular social choice function under investigation.

Definition 2: Let $m_{\mathrm{ij}}$ be the number of members preferring $a_{\mathrm{i}}$ to $a_{\mathrm{j}} ; m_{\mathrm{ji}}$ be the number of members preferring $a_{\mathrm{j}}$ to $a_{\mathrm{i}} ; m_{\mathrm{ij}}^{*}$ be the number of members being indifferent between $a_{\mathrm{i}}$ and $a_{\mathrm{j}}$. Then $\mathbf{M}$ is a proportion matrix whose elements represent the proportion of voters preferring one alternative to the other:

$M_{\mathrm{ij}}=\left(m_{\mathrm{ij}}+m_{\mathrm{ij}}^{*}\right) / n ; M_{\mathrm{ij}}=1 / 2 i \neq j$

Definition 3: Let $\mathbf{E}=\left(e_{\mathrm{ij}}\right)$ be a translated election matrix, $\mathbf{E}=\mathbf{M}-\mathbf{M}^{\mathrm{T}}$, where $\mathbf{M}^{\mathrm{T}}$ is the transpose of $\mathbf{M}$. The element $e_{\mathrm{ij}}$ represents the difference between the proportion of voters preferring $a_{\mathrm{i}}$ to $a_{\mathrm{j}}$ and those preferring $a_{\mathrm{j}}$ to $a_{\mathrm{i}}$.

Then the Kemeny function is defined to be:

$f_{\mathrm{K}}=\max \langle\mathbf{E}, \mathbf{L}\rangle$

where $\langle\mathbf{E}, \mathbf{L}\rangle$ is the ordinary inner product of $\mathbf{E}$ and $\mathbf{L}$; i.e., $\sum_{\mathrm{ij}} \mathrm{l}_{\mathrm{ij}} \mathrm{e}_{\mathrm{ij}}$

From many possible group ranking of alternatives, a particular group ranking is said to maximize the amount of agreements among group members when its Kemeny score; i.e., the value of $\langle E, L\rangle$, is the largest.

\section{AGGREGATION OF INDIVIDUAL PREFERENCES}

\section{The Decision Problem}

The decision problem relates to project selection and economic appraisal of proposals for new products in a manufacturing company by a Board of Directors having nine members. The decision problem (choice set) is presented in Table 1.

Table 1: Decision Problem

\begin{tabular}{|l|c|c|c|c|c|}
\hline & Project 1 & Project 2 & Project 3 & Project 4 & Project 5 \\
\hline -NPV of Cash Flow (in millions) & $\$ 3.5$ & $\$ 5.0$ & $\$ 1.5$ & $\$ 4.0$ & $\$ 2.5$ \\
-Initial Investment (in millions) & $\$ 1.5$ & $\$ 3.0$ & $\$ 0.5$ & $\$ 2.0$ & 1.0 \\
-Market Growth Rate & Fair & V.good & Good & Good & V.good \\
-Capability to Market & Good & Fair & V.good & Good & V.good \\
-Prospect of Technical Success & Fair & Fair & V.good & Good & good \\
\hline
\end{tabular}

There exist tradeoffs among criteria and no single criterion absolutely dominates any others. The proposals are rated on a scale from 0 to 100 , individually by nine board members. Individual preferences/utilities on each proposal were assessed with a procedure described in Keeney (1977). Then group rankings were aggregated from individual preferences/rankings from members. Table 2 presents the preference order of the choice set by group members.

Table 2: Preference Orders of 5 Projects by Group Members

\begin{tabular}{|c|c|c|c|c|c|c|c|c|c|}
\hline Member 1 & P3 & $>$ & P5 & $>$ & P4 & $>$ & P2 & $>$ & P1 \\
\hline Member 2 & P3 & $>$ & P5 & $>$ & P4 & $>$ & P2 & $>$ & P1 \\
\hline Member 3 & P3 & $>$ & P5 & $>$ & $\mathrm{P} 2$ & $>$ & P4 & $>$ & P1 \\
\hline Member 4 & P5 & $>$ & P3 & $>$ & P4 & $>$ & P2 & $>$ & P1 \\
\hline Member 5 & P5 & $>$ & P3 & $>$ & P4 & $>$ & P1 & $>$ & $\mathrm{P} 2$ \\
\hline Member 6 & P5 & $>$ & P4 & $>$ & P3 & $>$ & P1 & $>$ & $\mathrm{P} 2$ \\
\hline Member 7 & P5 & $>$ & P3 & $>$ & P4 & $>$ & P1 & $>$ & $\mathrm{P} 2$ \\
\hline Member 8 & $\mathrm{P} 2$ & $>$ & P5 & $>$ & P4 & $>$ & P3 & $>$ & P1 \\
\hline Member 9 & P4 & $>$ & P2 & $>$ & P5 & $>$ & P3 & $>$ & P1 \\
\hline
\end{tabular}




\section{Findings and Discussion}

Assuming equal member weights/powers, one can take either arithmetic mean or geometric mean of individual utilities for each project to derive at a group utility. The former rule averages out differences in individual preferences among group members. The later rule, called the Nash bargaining model, maximizes the product of individual utilities for each project over a "status quo"; i.e., a project with all being at worst.

Using the social welfare approach with arithmetic means to average individual utilities/preferences, the study arrived at the following group rankings.

$P 5>P 3>P 4>P 2>P 1$

Using the social choice function approach, one arrived at the following rankings:

$\begin{array}{llll}\text { Condorcet Function Ranking: } & f_{\mathrm{C}}(P 5)>f_{\mathrm{C}}(P 3)>f_{\mathrm{C}}(P 2)>f_{\mathrm{C}}(P 4)>f_{\mathrm{C}}(P 1) \\ \text { Borda Function Ranking: } & f_{\mathrm{B}}(P 5)>f_{\mathrm{B}}(P 3)>f_{\mathrm{B}}(P 4)>f_{\mathrm{B}}(P 2)>f_{\mathrm{B}}(P 1) \\ \text { Copeland Function Ranking: } & f_{\mathrm{CP}}(P 5)>f_{\mathrm{CP}}(P 3)>f_{\mathrm{CP}}(P 4)>f_{\mathrm{CP}}(P 2)>f_{\mathrm{CP}}(P 1) \\ \text { Eigenvector Function Ranking: } & f_{\mathrm{E}}(P 5)>f_{\mathrm{E}}(P 3)>f_{\mathrm{E}}(P 4)>f_{\mathrm{E}}(P 2)>>f_{\mathrm{E}}(P 1)\end{array}$

Scores and ranks of the choice set are presented in Table 3. Function scores are reported in the brackets. Kemeny scores for group ranking are calculated at the end of the table.

Table 3: Group Utility vs. Social Choice Functions Scores and Ranks

\begin{tabular}{|l|c|c|c|c|c|c|}
\hline & Group Utility & Condorcet & Borda & \multicolumn{2}{|c|}{ Copeland } & Eigenvector \\
\hline Project 1 & $(.503) 5$ & $(0) 5$ & $(3) 5$ & $(-4) 5$ & $(.030)$ & 5 \\
\hline Project 2 & $(.570) 4$ & $(2) 3$ & $(12) 4$ & $(-2) 4$ & $(.071)$ & 4 \\
\hline Project 3 & $(.741) 2$ & $(3) 2$ & $(25) 2$ & $(2) 2$ & $(.241)$ & 2 \\
\hline Project 4 & $(.652) 3$ & $(1) 4$ & $(20) 3$ & $(0) 3$ & $(.156)$ & 3 \\
\hline Project 5 & $(.763) 1$ & $(6) 1$ & $(30) 1$ & $(3) 1$ & $(.502)$ & 1 \\
\hline Kemeny & $(116 / 9)$ & $(96 / 9)$ & $(116 / 9)$ & $(116 / 9)$ & $(116 / 9)$ \\
\hline
\end{tabular}

Results show that social choice functions produce similar group ranking from those obtained with social welfare functions. This implies that one may not need complex assessment methods for cardinal preferences of social welfare approach. Even the implementation of complex counting rules such as the Eigenvector function may not be necessary.

This study is based on the assumptions of equal weights among group members. However, member weights - voting powers - could be incorporated in the social choice function. Instead of assessing utility tradeoffs among group members, a group can easily decide on the number of extra votes for some key members. For example, in this study, the group may agree that Member A can cast two votes. Then, the group will function as if there are ten members and the aggregation process will account for two duplicate rankings from Member A.

\section{CONCLUSION}

This study finds the similarity among social choice functions and the comparable performance of the simple function against the complex ones. It empirically confirms the ability on Copeland function as a simple and accurate aggregation rule for a Group Decision Support System.

This study works on the individual preference assessed by cardinal utility function with a procedure described in Keeney (1977). Elsewhere we discuss evidences supporting a holistic preference approach as an alternative to utility-based assessment to overcome the difficulty of decision makers in expressing explicitly their decision rules and their heuristics in the preference assessment process. 


\section{AUTHOR INFORMATION}

Dat-Dao Nguyen, Ph.D., is a Professor of Information Systems in Department of Accounting and Information Systems at California State University - Northridge. He holds a PhD degree in MIS from Concordia University (Montreal, Canada). His researches have been published in IEBM Handbook of Information Technology in Business, The International Encyclopedia of Business and Management, The Internet Encyclopedia, Journal of Information Science and Technology, Journal of College Teaching \& Learning, Contemporary Issues In Education Research, and Proceedings of Decision Sciences Institute, among others. Dat- Dao Nguyen, Ph.D., California State University, 18111 Nordhoff Street, Northridge, CA 91330-8372 USA. E-mail: datdao.nguyen@csun.edu

\section{REFERENCES}

1. Ben-Arieh D., \& Easton T. (2007). Multi-criteria group consensus under linear cost opinion elasticity. Decision Support Systems, 43(3), 713-721.

2. Cook, W. D., \& Seiford, L. M. (1982). On the Borda-Kendall consensus methods for priority ranking problems. Management Science, 28(6), 621-637.

3. DeSanctis \& Minnesota GDSS Research Group (2008). The Minnesota GDSS Research project: Group Support Systems, Group Processes, and Outcomes. Journal of the Association for Information Systems, 9(10/11), 551-608

4. Felsental, D. S., \& Machover, M. (1992). After two centuries, should Condorcet's voting procedure be implemented? Behavior Science, 37, 250-274.

5. Hwang, C. L., \& Lin, M. J. (1987). Group decision making under multiple criteria: Methods and applications. Springer-Verlag, Berlin.

6. Keeney, R. L. (1976) A group preference axiomatization with cardinal utility. Management Science, 23(2), 140-145.

7. Keeney, R. L. (1977). The art of assessing multiattribute utility functions. Organizational Behavior and Human Performance, 19, 267-310.

8. Keeney, R. L., \& Kirkwoods, C. W. (1975). Group decision making using cardinal social welfare function. Management Science, 22(4), 430-437.

9. Kemeny, J. G., \& Snell, J. L. (1962). Mathematical models in the social sciences. Boston: Ginn and Co.

10. Levenglick, A. (1975). Fair and reasonable election systems. Behavior Science, 20, 34-46.

11. Richelson, J. (1975). A comparative analysis of social choice functions. Behavior Science, 20, 331-337.

12. Richelson, J. (1978a). A comparative analysis of social choice functions, II. Behavior Science, 23, 38-44.

13. Richelson, J. (1978b). A comparative analysis of social choice functions, III. Behavior Science, 23, 169170.

14. Richelson, J. (1981). A comparative analysis of social choice functions, IV. Behavior Science, 26, 346-353.

15. Turban, E., Sharda R., \& Delen D. (2011). Decision support and business intelligent systems $\left(9^{\text {th }}\right.$ Ed.). Upper Saddle River, NJ: Prentice-Hall.

16. Wallsten, T. S., \& Budescu, D. V. (1983). Encoding subjective probabilities: A psychological and psychometric review. Management Science, 29(2), 151-173. 